\title{
Kikuchi-Fujimoto Disease Coexistent with Papillary Thyroid Carcinoma in a Single Lymph Node
}

\author{
Jin Ju Park', Yu Bin Seo ${ }^{1}$, Hyun Chang Choi', Jeong Won Kim², Mi Kyung Shin², Dong Jin Lee ${ }^{3}$, Jacob Lee ${ }^{1}$ \\ Departments of 'Internal Medicine, ${ }^{2}$ Pathology, and ${ }^{3}$ Otorhinolaryngology-Head and Neck Surgery, Hallym University College of Medicine, Chuncheon, Korea
}

\begin{abstract}
Cervical lymphadenopathy can be developed from various causes such as viral infection, bacterial infection, Kikuchi-Fujimoto disease, tuberculosis, malignancy, and reactive changes. In patients who have malignancy, metastatic lymphadenopathy is possible but it is rare that other concomitant diseases are in the same lymph node. We experienced a case of coexistence of Kikuchi necrotizing lymphadenitis and papillary thyroid carcinoma in a single cervical lymph node. A 38-year-old man who was previously diagnosed with papillary thyroid cancer with cervical lymph nodes metastasis presented with cervical lymphadenopathy. The lymph node biopsy showed tuberculous lymphadenitis. After finishing anti-tuberculosis medication, recurrent lymphadenopathy had developed and a surgical biopsy was performed. At that time, the diagnosis was Kikuchi necrotizing lymphadenitis combined with metastatic papillary carcinoma in a single lymph node.
\end{abstract}

Keywords: Lymphadenopathy; Biopsy; Histiocytic necrotizing lymphadenitis; Tuberculous lymphadenitis; Papillary thyroid carcinoma; Coexistent disease

\section{INTRODUCTION}

Cervical lymphadenopathy can be developed from various causes such as viral infection, bacterial infection, Kikuchi-Fujimoto disease (KFD), tuberculosis (TB), malignancy, and reactive changes [1]. Among these, KFD is a benign, self-limited syndrome characterized by cervical lymphadenopathy, fever and malaise that occurs predominantly in young people [2]. The etiology of KFD is unclear. The association of KFD and malignancy is also seldom discussed.

In patients who have malignancy, metastatic lymphadenopathy is possible. But it is rare that other concomitant diseases are in the same lymph node [3-9]. There have been some case reports related to concomitant two other diseases in a lymph node $[3,5,8,10]$. To our best knowledge, however, there is no case report of KFD and papillary thyroid carcinoma combined in a single lymph node. Here, we report a case of a 38-year-old man who has undergone surgical biopsy for recurrent cervical lymphadenopathy and were diagnosed with coexisted KFD and thyroid cancer in a lymph node.

\section{CASE REPORT}

A 38-year-old man was admitted on July 4, 2010 with a history of gradually worsening right cervical lymph node enlargement for a week. He did not have any past medical history and take medicine. He did not present any constitutional symptoms. On physical examination, non-tender, fixed lymph nodes were palpable at the right posterior neck. White blood cell count (WBC) was $2,550 / \mu \mathrm{L}$ (normal value, 4,000 to $8,000 / \mu \mathrm{L}$ ), platelet count was $203 \times 10^{3} / \mu \mathrm{L}$ (normal value, 150 to $450 \times 10^{3} / \mu \mathrm{L}$ ), lymphocyte proportion was $51.4 \%$ (normal value, $19 \%$ to $48 \%$ ), aspartate aminotransferase, alanine aminotransferase was normal with 20 IU/L (normal value, 9 to $39 \mathrm{IU} / \mathrm{L}$ ), $22 \mathrm{IU} / \mathrm{L}$ (normal value, 5 to $45 \mathrm{IU} / \mathrm{L}$ ), and C-reactive peptide (CRP) was $1.8 \mathrm{mg} / \mathrm{L}$ (normal value, 0 to $3.0 \mathrm{mg} / \mathrm{dL}$ ). Free $\mathrm{T} 4$ was $1.10 \mathrm{ng} / \mathrm{dL}$ (normal value, 0.89 to $1.76 \mathrm{ng} / \mathrm{dL}$ ), thyroid-stimulating hormone (TSH) was $2.59 \mu \mathrm{IU} / \mathrm{mL}$ (normal value, 0.550 to $4.780 \mu \mathrm{IU} / \mathrm{mL}$ ), and thyroglobulin (Tg) was $1.2 \mathrm{ng} / \mathrm{mL}$ (normal value, 0 to $35 \mathrm{ng} / \mathrm{mL}$ ). Contrast enhanced neck computer tomography
Correspondence to: Jacob Lee

Division of Infectious Disease, Department of Internal Medicine, Hallym University Kangnam Sacred Heart Hospital, Hallym University College of Medicine, 1 Singil-ro, Yeongdeungpo-gu, Seoul 150-950, Korea

Tel: +82-2-829-5107, Fax: +82-2-6918-4222, E-mail: litjacob@chol.com

Received: Dec. 5, 2014 / Accepted after revision: Apr. 7, 2015
(C) 2015 Soonchunhyang Medical Research Institute This is an Open Access article distributed under the terms of the Creative Commons Attribution Non-Commercial License (http://creativecommons.org/licenses/by-nc/3.0/) 
(CT) showed 2- to 6-mm-sized, round, multiple hypodense lesions in the right upper thyroid lobe and 4- to 10 -mm-sized, irregular, rim enhanced multiple enlarged lymph nodes in ipsilateral internal jugular chain (IJC) (Fig. 1A, B). Fine needle aspiration (FNA) was done targeting the right thyroid mass. The pathologic finding was papillary thyroid carcinoma on thyroid mass. Total thyroidectomy and central neck dissection (CND) were performed and multifocal papillary thyroid carcinoma with lymph node metastasis (stage III, T2bN1aM0) was diagnosed (Fig. 1C). He took radioactive iodine therapy and had been treated with levothyroxine 0.2 mg. Eight months after the surgery, follow-up ${ }^{131}$ I whole body scan did not show any hot uptake and $\mathrm{Tg}$ was within normal range.

One year after the total thyroidectomy with $\mathrm{CND}$, the patient started to complain of intermittent fever up to $38.3^{\circ} \mathrm{C}$ with chill, dry cough, and night sweat. On physical examination, about 1-cm-sized right supraclavicular lymph node was palpable and no other abnormal findings were found. The lymph node was non- tender and firm and was presented with overlying erythema. The laboratory data showed elevated CRP as $64.7 \mathrm{mg} / \mathrm{L}$ and normal $\mathrm{WBC}$ as $4,300 / \mu \mathrm{L}$. Thyroid profile was normal as the following results: free T4 $1.60 \mathrm{ng} / \mathrm{dL}$ and TSH $1.67 \mu \mathrm{IU} / \mathrm{mL}$. There was no abnormal finding with the chest X-ray. On chest CT, multiple lymph nodes enlargement appeared in the right supraclavicular, right paratracheal, and subcarinal area. The lymph nodes were 4- to 14-mm-sized, round-shaped, and peripheral rim enhanced with central necrosis. 18F-fluorodeoxyglucose (FDG) positron emission tomography (PET)/CT was done in which there were hypermetabolic lymph nodes in right neck, supraclavicular, and mediastinal area (Fig. 2A). FNA on right IJC lymph node was done and just atypical cells were reported. Because the central necrosis was more prominent in mediastinal area on CT image, paratracheal lymph nodes biopsy was done by a thoracic surgery doctor for definite diagnosis. Through microscopic examination, chronic granulomatous inflammation with caseous necrosis was observed.
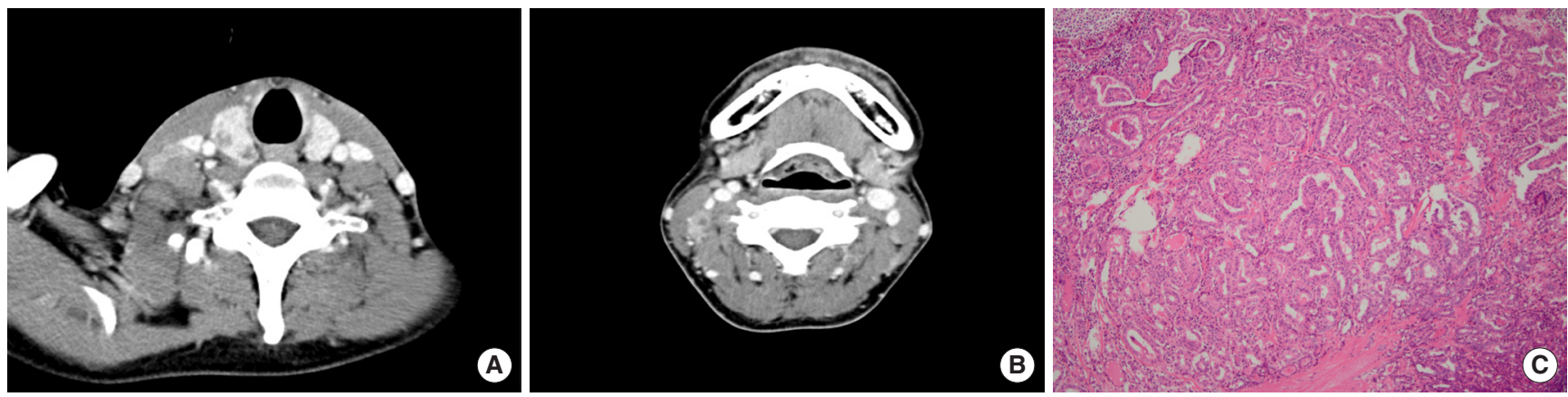

Fig. 1. Contrast-enhanced neck computed tomography scan showed (A) multiple hypodence lesions in upper pole of right thyroid gland and (B) internal jugular chain lymph node enlargement with central necrosis. (C) The excised anterior central neck dissection specimen revealed metastasis to three central lymph nodes (H\&E, $\times 100)$.
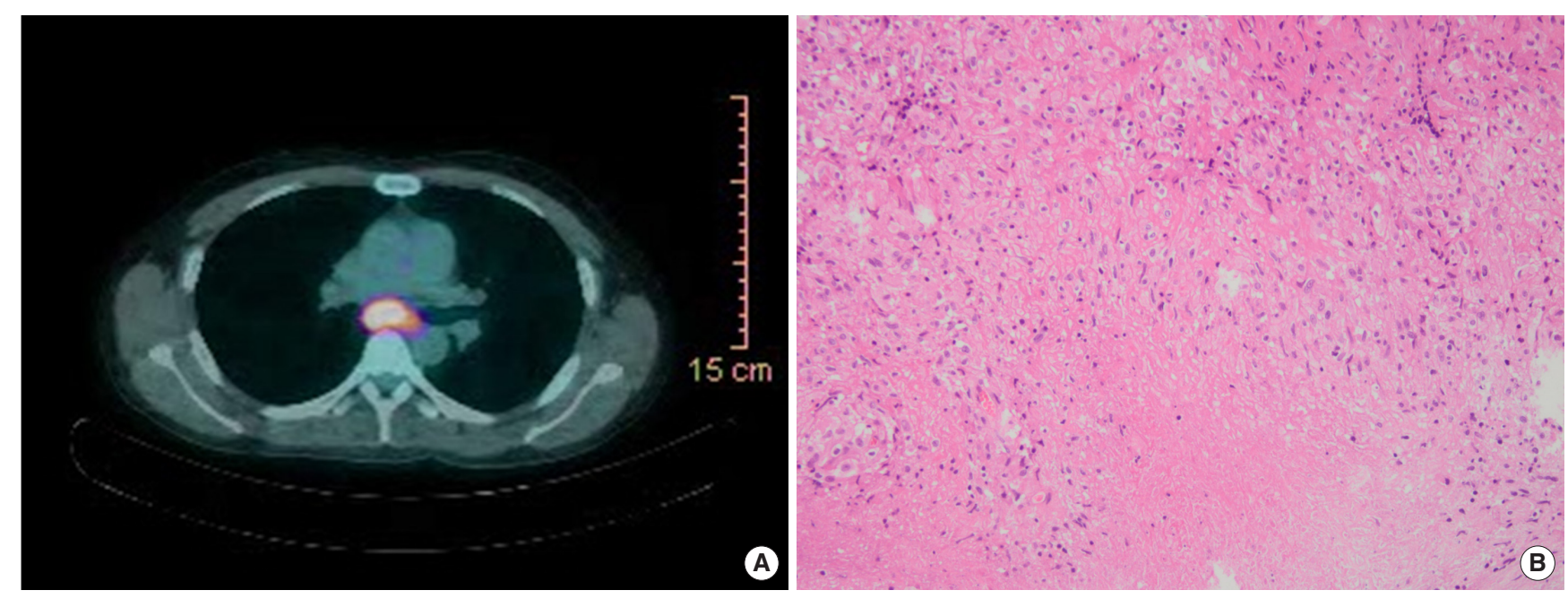

Fig. 2. (A) 18F-fluorodeoxyglucose positron emission tomography/computed tomography showed hypermetabolic uptake on mediastinal and supraclavicular area. (B) Microscopically, enlarged paratracheal lymph nodes showed caseating granulomatous inflammation, consistent with tuberculosis $(H \& E, \times 400)$. 
Park JJ, et al. • Coexistent Kikuchi-Fujimoto Disease and Papillary Thyroid Carcinoma

This was consistent with TB (Fig. 2B). The result of nested polymerase chain reaction (PCR) for mycobacterium tuberculosis was also positive. He started anti-TB medication made up of isoniazide, rifampin, pyrazinamide and ethambutol. After six months of treatment, right lower neck lymph nodes were more enlarged on physical examination despite the anti-TB therapy. Considering the paradoxical response, anti-TB therapy was continued further three months. After finishing the anti-TB therapy for nine months, he took 18F-FDG PET/CT. Preexisting lymph nodes on left paratracheal disappeared, and lymph nodes on the right supraclavicular, right paratracheal, and subcarinal area decreased in size by about half to one third.

Nine months after the end of the anti-TB therapy, he visited the hospital due to fever, dry cough, sore throat, and generalized myalgia for two weeks. With the physical exam, about $1.5-\mathrm{cm}$-sized
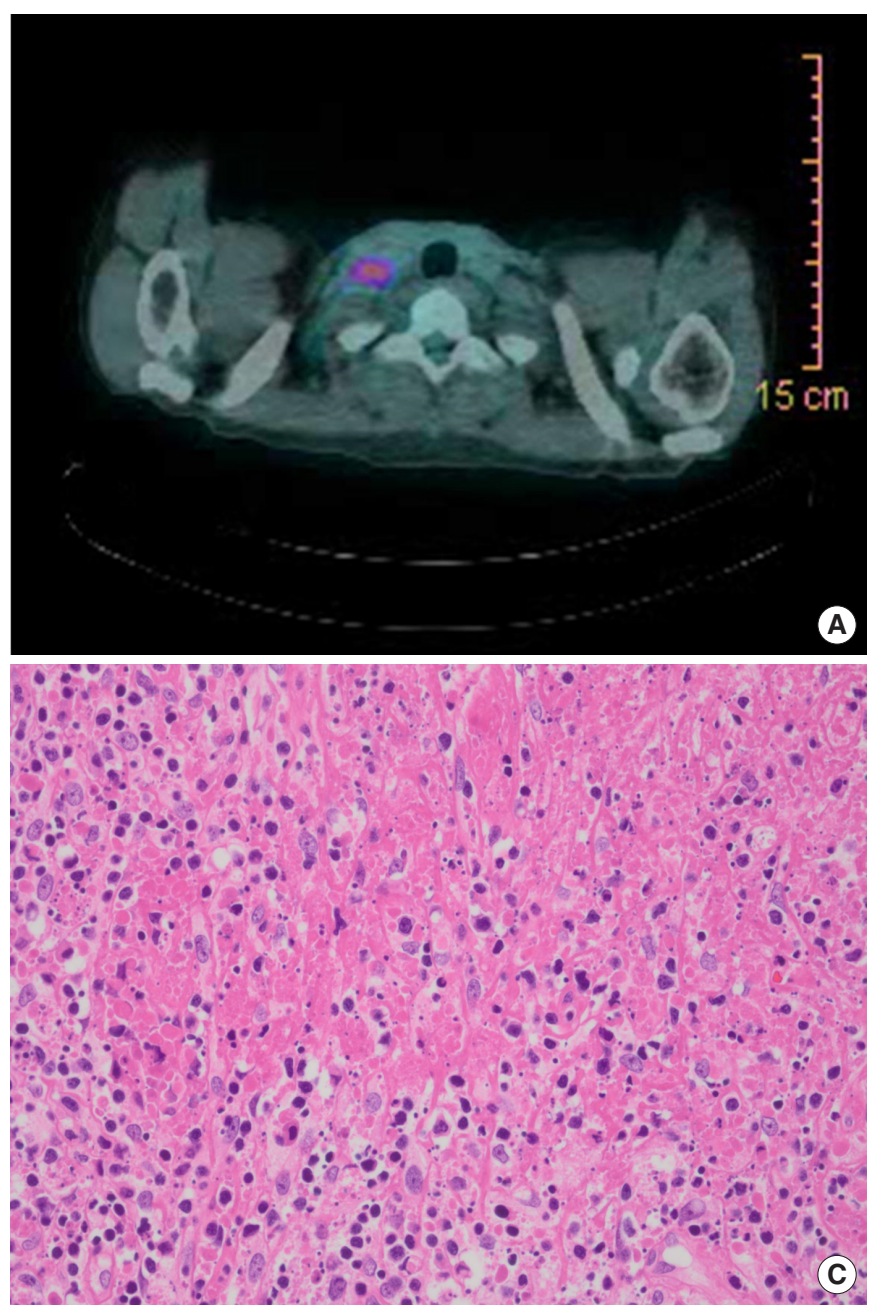

firm, fixed, and tender lymph node was newly palpable on the right posterior neck. Laboratory reports were as follows: WBC $2,760 / \mu \mathrm{L}$, CRP 33.3 mg/L, TSH $0.007 \mu \mathrm{IU} / \mathrm{mL}$, free T4 $1.48 \mathrm{ng} / \mathrm{dL}$, and Tg $<0.100 \mathrm{ng} / \mathrm{mL}$. On 18F-FDG PET/CT, right supraclavicular, paratracheal, and subcarinal lymph nodes increased comparing with the previous 18F-FDG PET/CT. In addition, both axillary and retroperitoneal lymph nodes enlargements were newly observed (Fig. 3A). Excision biopsy was done on the right cervical and right supraclavicular lymph node. Microscopically, the lymph nodes were composed of focal, well circumscribed subcortical necrotizing lesions with abundant karyorrhectic debris, compatible with KFD (Fig. 3B, C). On immunohistochemical stain, there were many histiocytes with immunoreactivity for CD68 and myeloperoxidase. Simultaneously, microscopic foci of metastatic papillary carcinoma were identified in the same lymph node (Fig. 3B, D). TB was not
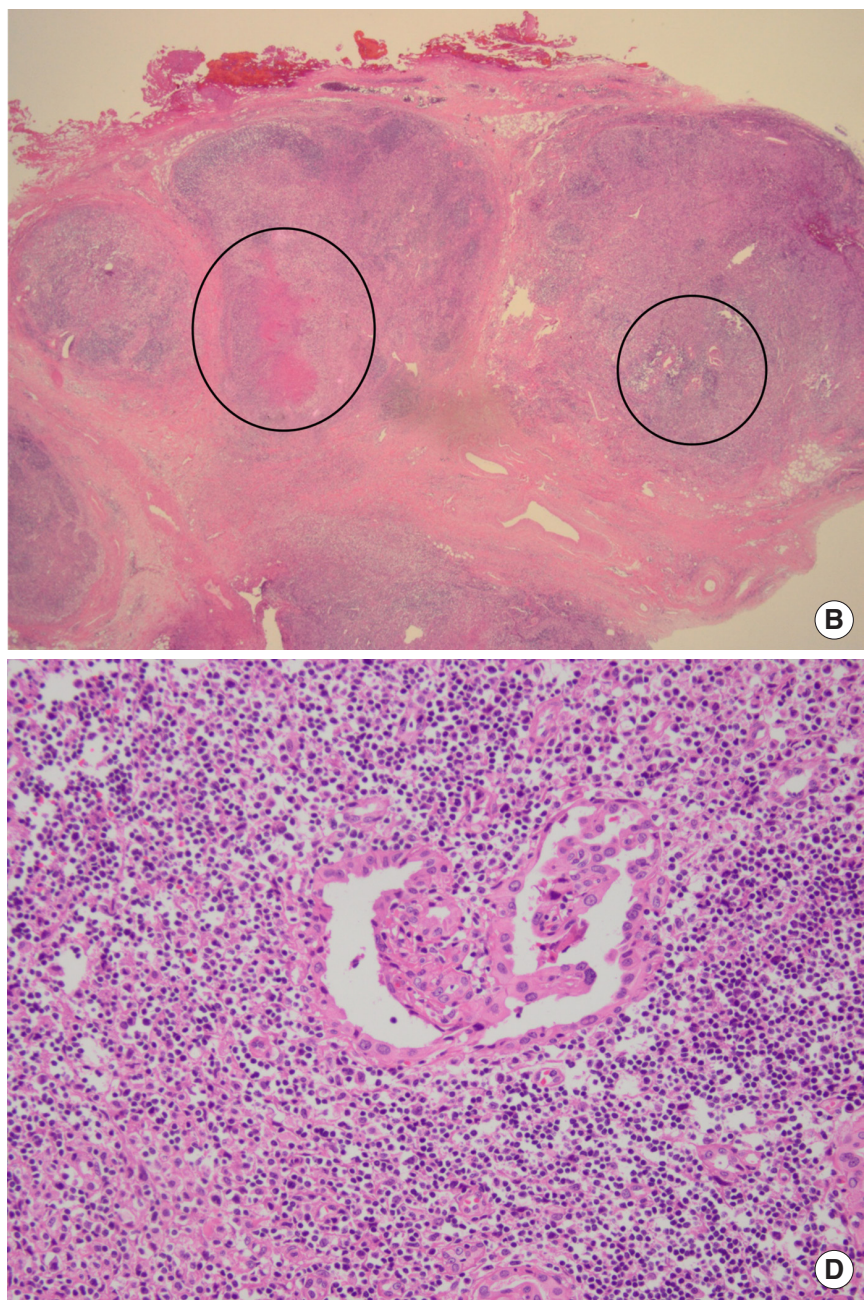

Fig. 3. In 18F-fluorodeoxyglucose positron emission tomography/computed tomography image (A) right supraclavicular lymph node was increased uptake. (B) In one supraclavicular lymph node $(\mathrm{H} \& \mathrm{E}, \times 40)$, (C) there were abundant karyorrhectic debris, fibrin deposits and scattered mononuclear cells, compatible histologic findings of Kikuchi necrotizing lymphadenitis (H\&E, $\times 1,000)$. (D) A microscopic focus of metastatic papillary carcinoma was coincidentally identified in the lymph node (H\&E, $\times 400)$. 
suspected in microscopic finding and TB nested PCR was negative. The final diagnosis was KFD combined with metastatic papillary carcinoma. Because the malignant pathologic finding was just a small portion of lymph node and he already had taken a total thyroidectomy and lymph node dissection, we decided to continue the levothyroxine medication without further radioactive iodine therapy or operation under the discussion with endocrinologist. Instead, methylprednisolone and non-steroidal anti-inflammatory drugs were added to treat the KFD. Three months later, hypermetabolic lymph nodes at mediastinal and axillary area were completely diminished in 18F-FDG PET/CT and his symptoms have been completely resolved without recurrent symptoms.

\section{DISCUSSION}

Metastatic lymphadenopathy is possible in patients with malignancy. But it is rare that other concomitant diseases are in the same lymph node [3-9]. The case of lymphoma and localized Castleman' $s$ disease within the same lymph node was reported [11]. This could be possible because localized Castleman's disease has potential to develop into lymphoma, sarcoma, and carcinoma. Also the coexistence of TB and malignancy such as lymphoma, leukemia, and solid tumor has been discussed before [5]. This can be possible due to the impaired cell-mediated immunity that is affected by immunosuppressive therapy or malignancy itself. This coexistence can be possible subsequently. Incidence of TB in patients who have lymphoma is much higher than general population [12].

KFD is a self-limited and benign disease characterized by cervical lymphadenopathy, fever, and malaise. The etiology of KFD is unclear but some have reported infectious and autoimmune etiologies [2]. It means that KFD can occur more frequently under immunosuppressive condition like TB. But there is no accurate reported study about mechanism and relationship.

Our case is about a patient who had twice-recurrent cervical lymphadenopathy. Initially, the thyroidectomy and neck dissection revealed them as metastatic papillary carcinoma. One year after the surgery, he complained of cervical lymphadenopathy and fever. Because he had thyroid cancer history, the possibility of recurrence could not be ruled out. Through the surgical biopsy, he was diagnosed with TB lymphadenitis. After the anti-TB therapy, cervical lymphadenopathy was unfortunately developed again. Surgical biopsy confirmed the final diagnosis as KFD accompanied by metastatic papillary thyroid cancer. Interestingly, KFD and metastatic thyroid cancer coexisted in one lymph node and twice-recurrent cervical lymphadenopathy were diagnosed differently from each other. The pathology could be the results of remained thyroid cancer or incidental finding. However, it might be meaningful case. Studies and cases about researching cause of KFD have been reported but mostly limited to virus or autoimmune disease. As mentioned, under the immunosuppressive condition virus may activate KFD more frequently. However only one case have been reported the coexistent KFD and carcinoma until now [13]. The association between KFD and malignancy is obscure and there is no reported study. Our case suggests the need for further studies to reveal the relationship of KFD and malignancy as well as KFD and TB. In addition, which treatment should be chosen is another dilemma. Decision to treat both two disease or not is always considered and wise choice is needed.

The coexistence of two other diseases is rare but diseases that the association have not been unproven can coexist, the possibility of other concurrent diseases have always to be kept in mind especially in patients with malignancy history.

\section{REFERENCES}

1. Song JY, Cheong HJ. Diagnosis and treatment of cervical lymphadenitis from a standpoint of infectious disease specialists. Infect Chemother 2008;40:76-82.

2. Bosch X, Guilabert A. Kikuchi-Fujimoto disease. Orphanet J Rare Dis 2006;1:18.

3. Bellido MC, Martino R, Martínez C, Sureda A, Brunet S. Extrapulmonary tuberculosis and non-Hodgkin's lymphoma: coexistence in an abdominal lymph node. Haematologica 1995;80:482-3.

4. Carbone A, Volpe R. Kaposi's sarcoma in lymph nodes concurrent with Hodgkin's disease. Am J Clin Pathol 1983;80:228-30.

5. Centkowski P, Sawczuk-Chabin J, Prochorec M, Warzocha K. Hodgkin’s lymphoma and tuberculosis coexistence in cervical lymph nodes. Leuk Lymphoma 2005;46:471-5.

6. Fanourgiakis P, Mylona E, Androulakis II, Eftychiou C, Vryonis E, Georgala A, et al. Non-Hodgkin's lymphoma and tuberculosis coexistence in the same organs: a report of two cases. Postgrad Med J 2008;84:276-7.

7. Panikar N, Sikka M, Singh N. Concurrent chronic myelogenous leukemia and tuberculous lymphadenitis: a case report. Acta Cytol 2005;49: 650-2.

8. Selvasekar A, Ebenezer GJ, Partheebarajan M. Lepromatous lymphadenopathy and concomitant tuberculous axillary lymphadenitis with sinus: a case report. Lepr Rev 1999;70:345-50.

9. Kakarala K, Sadow PM, Emerick KS. Cervical lymph node collision tumor consisting of metastatic squamous cell carcinoma and B-cell lymphoma. Laryngoscope 2010;120 Suppl 4:S156.

10. Gaurav M, Rajgopal S, Mary M, Bhavatej E. Concomitant tuberculosis and metastasis in axillary lymph nodes: a diagnostic surprise. ANZ J Surg 2008;78:825-6.

11. Orcioni GF, Mambelli V, Ascani S, Sabattini E, Piccioli M, Pieri F, et al. 
Park JJ, et al. • Coexistent Kikuchi-Fujimoto Disease and Papillary Thyroid Carcinoma

Concurrence of localized Castleman's disease and peripheral small B-Lymphocytic lymphoma within the same lymph node. Gen Diagn Pathol 1998; 143:327-30.

12. Ruiz-Arguelles GJ, Mercado-Diaz MA, Ponce-De-Leon S, Ponce-De-Le- on S, Perez-Tamayo R. Studies on lymphomata: III. lymphomata, granulomata and tuberculosis. Cancer 1983;52:258-62.

13. Radhi JM, Skinnider L, McFadden A. Kikuchi’s lymphadenitis and carcinoma of the stomach. J Clin Pathol 1997;50:530-1. 